\title{
Proliferation and differentiation of direct co-culture of bone marrow mesenchymal stem cells and pigmented cells from the ciliary margin
}

\author{
YAN LI ${ }^{1}$, XINZHENG HE $^{1}$, JUN LI ${ }^{1}$, FANGFANG NI ${ }^{1}$, QINGQING SUN ${ }^{1}$ and YAN ZHOU ${ }^{2}$ \\ ${ }^{1}$ Department of Ophthalmology, No. 113 Hospital of PLA; ${ }^{2}$ Department of Glaucoma, \\ Ningbo Eye Hospital, Ningbo, Zhejiang 315000, P.R. China
}

Received February 14, 2016; Accepted February 7, 2017

DOI: $10.3892 / \mathrm{mmr} .2017 .6481$

\begin{abstract}
Damage of retinal ganglion cells (RGCs) is the major consequence of glaucoma and regeneration of RGCs is extremely difficult once the damage has occurred. Retinal stem cells (RSCs) are considered an ideal choice for RGC regeneration. Pigmented cells from the ciliary margin (PCMs) have great retinal differentiation potential and may be an ideal RSC candidate. However, the ciliary margin is too small, so the number of cells that can be obtained is limited. Bone marrow-derived mesenchymal stem cells (BMMSCs) are another type of stem cell that have been previously investigated for RGC regeneration. BMMSCs expand sufficiently, whereas the retinal differentiation of BMMSCs is insufficient. The aim of the present study was to investigate whether the co-culture of PCMs and BMMSCs may combine the advantages of both cell types to establish a novel and effective stem cell source for RGC regeneration. Primary rat PCMs and BMMSCs were isolated and co-cultured. Cell growth was observed by an inverted microscope and proliferation was monitored by an MTT assay. Cell cycle analysis was performed by using a flow cytometer, while the expression of the photoreceptor-specific homeobox gene (cone-rod homeobox, Crx) was determined by reverse transcription-quantitative polymerase chain reaction and western blot analysis. In addition, retinal differentiation was confirmed by immunofluorescence staining of major markers of retinal differentiation, including rhodopsin, visual system homeobox 2 and heparin sulfate. The co-cultured cells expanded successfully, in a similar way to BMMSCs. In addition, the expression of $\mathrm{Crx}$ and retinal markers were significantly upregulated following BMMSC and PCM co-culture. The results of the present study demonstrated that
\end{abstract}

Correspondence to: Dr Yan Zhou, Department of Glaucoma, Ningbo Eye Hospital, 855 Min'an Road, Ningbo, Zhejiang 315000, P.R. China

E-mail: yanzhou1978@hotmail.com

Key words: glaucoma, retinal stem cells, pigmented cells from the ciliary margin, bone marrow mesenchymal stem cells, co-culture the co-culture of BMMSCs and PCMs may be used as a source of RSCs.

\section{Introduction}

Glaucoma is one of the most prevalent eye diseases and is characterized by gradual sight field loss that is caused by damage to the optical nerve (1). As the optical nerve is considered to be the central nerve system among cranial nerves, it is almost impossible for spontaneous regeneration to occur once the impairment has occurred (2). Once the death of retinal ganglion cells (RGCs) has occurred, it cannot be reversed. The majority of patients already have some RGC impairment when seeking medical help and nothing can currently be done to reverse the progress. Therefore, the ultimate goal for glaucoma treatment is the regeneration of RGCs (3).

With the development of stem cell technology, the regeneration of RGCs has been investigated in depth. An ideal stem cell type for retina regeneration may be retinal stem cells (RSCs), a group of stem cells in the optic vesicle, which generate all the neurons and the Müller glia of the mature retina (4). In adult mammalian eyes, RSCs degenerate but remain in the ciliary margin area, the outer contour of the mature retina architecture (2). In 2000, Tropepe et al (5) first reported the identification RSCs in the adult mouse eye, which were subsequently named pigmented cells from the ciliary margin (PCMs). This result was important for retina regeneration through stem cell grafting, as the cells can be isolated from adult patients themselves and ethical issues, including the risk associated with using incompatible tissues, can be avoided. However, the adult mammalian ciliary margin is small and the proliferation ability of PCMs is also limited. As a result, the number of RSCs obtained from the PCM would be too low to utilize.

In addition, stem cells from other sources, including embryonic stem cells, fetal stem cells, umbilical tissue-derived stem cells, bone marrow-derived hematopoietic stem cells and bone marrow-derived mesenchymal stem cells (BMMSCs) have been extensively investigated (6). However, these stem cells originate from other types of tissue, so the retinal differentiation of these cells is limited. BMMSCs are one of the most prevalent stem cell sources used during tissue engineering due 
to the following properties: Strong multipotent differentiation ability, convenient accessibility and immunomodulatory functions (7). Although previous studies have revealed that BMMSCs migrate into the retinal region to repair the retina when injected intravenously, the retinal differentiation ability of BMMSCs remains limited $(8,9)$.

In the current study, the authors hypothesized that the combination of BMMSCs and PCMs may provide a novel stem cell group with improved retinal differentiation and proliferation capacities, with the potential to be a novel source of RSCs. Primary BMMSCs and PCMs were isolated from rats and the co-culture was performed by mixing the two cell types directly. Proliferation and cell cycle was investigated and the expression of retinal markers was observed to evaluate the potential for co-cultured cells to differentiate into retinal cells.

\section{Materials and methods}

Cell isolation and culture. PCMs and BMMSCs were isolated according to previous reports $(5,10)$ under the approval of the Research Ethics Committee of the School of Medicine, Ningbo University (Ningbo, China) and adhered to the Declaration of Helsinki for the use of animals in research. Briefly, male Sprague Dawley rats ( $n=5$; age, 6-8 weeks; weight, 200-250 g) were purchased from the School of Medicine, Ningbo University, and housed separately at $20-25^{\circ} \mathrm{C}$ under a 12 -h light/dark cycle and fed ad libitum with a normal diet. Rats were sacrificed by cervical dislocation and their eyes were harvested in oxygenated artificial cerebral spinal fluid $(124 \mathrm{mM} \mathrm{NaCl}, 5 \mathrm{mM} \mathrm{KCl}$, $1.3 \mathrm{mM} \mathrm{MgCl}_{2}, 2 \mathrm{mM} \mathrm{CaCl}_{2}, 26 \mathrm{mM} \mathrm{NaHCO}$ and $10 \mathrm{mM}$ $\mathrm{D}$-glucose). The whole ciliary margin was isolated and treated with dispase for $10 \mathrm{~min}$ at $37^{\circ} \mathrm{C}$. Tissue was subsequently dissected and placed in a trypsin solution at $37^{\circ} \mathrm{C}$ for $10 \mathrm{~min}$. Following this, cells were centrifuged at $150 \times \mathrm{g}$ for $5 \mathrm{~min}$ at room temperature and the enzyme solution was removed and replaced with serum-free media containing trypsin inhibitor (1 mg/ml ovomucoid; Roche Diagnostics, Basel, Switzerland), for $30 \mathrm{~min}$ at room temperature. Dissociated cells were plated in serum-free Dulbecco's Modified Eagle's medium/F12 (HyClone; GE Healthcare Life Sciences, Logan, UT, USA) supplemented with N-2 MAX (Thermo Fisher Scientific, Waltham, MA, USA) and cultured at $37^{\circ} \mathrm{C}, 5 \% \mathrm{CO}_{2}$ in a humidified atmosphere. PCM sphere colonies were formed following $\sim 1$ week of incubation. Meanwhile, the limbs of rats were separated and bone marrow was flushed out by douching with $\alpha$-minimal essential medium ( $\alpha$-MEM; HyClone; GE Healthcare Life Sciences) into the bone marrow cavity with a sterilized syringe. Aspirates were centrifuged at $150 \times g$ for $5 \mathrm{~min}$ at room temperature, resuspended and cultured in $\alpha$-MEM with $10 \%$ fetal bovine serum and $1 \%$ penicillin/streptomycin (all obtained from HyClone; GE Healthcare Life Sciences). Media was changed every 3 days and cells from passages 3-5 were used. For co-culture experiments, primary PCMs were pre-cultured in six-well plates at a density of $\sim 3 \times 10^{5}$ cells/well in serum-free DMEM/F12 + N-2 MAX for $24 \mathrm{~h}$ and then $\sim 2.5 \times 10^{5}$ BMMSCs were added. PCMs and BMMSCs were incubated in low serum (1\%) DMEM/F12 + N-2 MAX for further analysis. Following 3 days of incubation, the growth of cells was observed by inverted microscope (Olympus Corporation, Tokyo, Japan).
Proliferation analysis. The cell viability was monitored daily by MTT (MP Biomedicals, LLC, Santa Ana, CA, USA) as previously described (11). Briefly, cells were plated into 96-well plates at a concentration of 1,000 cells/well in $100 \mu 1$ medium. To assess viability, the medium was exchanged for an MTT working solution ( $5 \mathrm{mg} / \mathrm{ml}$ in cell culture medium) and incubated for $4 \mathrm{~h}$ at $37^{\circ} \mathrm{C}$. The solution was gently removed and the precipitation was dissolved in $100 \mu \mathrm{l}$ dimethylsulfoxide (MP Biomedicals, LLC), and measured at $490 \mathrm{~nm}$ with a spectrophotometer (BioTek Instruments, Inc., Winooski, VT, USA). The proliferation curve was plotted as optical density vs. culture time.

Cell cycle analysis. To further investigate cell division, the cell cycle was analyzed by flow cytometry, using a BD Accuri ${ }^{\mathrm{TM}}$ C6 (BD Biosciences, Franklin Lakes, NJ, USA). Briefly, following 3 days of culture, cells were detached by trypsin and washed with PBS to obtain a single cell suspension. Cells in the suspension were fixed with ice-cold dehydrated ethanol (absolute ethanol: PBS ratio, 2:3) overnight at $4^{\circ} \mathrm{C}$. The fixed cells were washed twice with PBS and stained by $100 \mathrm{mg} / \mathrm{ml}$ propidium iodide (PI; Sigma-Aldrich; Merck KGaA, Darmstadt, Germany) at $4^{\circ} \mathrm{C}$ for $30 \mathrm{~min}$. A total of $1 \times 10^{5}$ cells were collected for each sample and the PI-elicited fluorescence of individual cells was measured by flow cytometry. The amount of cells in the G0/G1 phase, $\mathrm{S}$ phase and G2/M phase was determined. Proliferation index was calculated as the percentage of cells in $\mathrm{S}+\mathrm{G} 2 / \mathrm{M}$.

Reverse transcription-quantitative polymerase chain reaction (RT-qPCR) analysis. Following expansion for 7 days in growth medium, retinal differentiation was performed using induction medium, which contained DMEM/F12 supplemented with N-2 MAX, 1\% FBS, $10 \mathrm{ng} / \mathrm{ml}$ fibroblast growth factor 2 (Prospec-Tany Technogene, Ltd. East Brunswick, NJ, USA) and $2 \mu \mathrm{g} / \mathrm{ml}$ heparin (Hebei Changshan Biochemical Pharmaceutical Co., Ltd., Hebei, China). Cells were cultured at $37^{\circ} \mathrm{C}$ and $5 \% \mathrm{CO}_{2}$ in a humidified atmosphere. Following 21 days of induction, cells were rinsed with PBS and total RNA was extracted by TRIzol reagent (Invitrogen; Thermo Fisher Scientific, Inc.). Total RNA (1 $\mu \mathrm{g})$ was reverse transcribed into cDNA by using the qScript cDNA SuperMix kit (Quanta Biosciences, Beverly, MA, USA) according to the manufacturer's protocol. This was performed on Bio-Rad CFX (Bio-Rad Laboratories, Inc., Hercules, CA. USA) with Platinum ${ }^{\circledR}$ SYBR ${ }^{\circledR}$ Green qPCR SuperMix-UDG (Invitrogen; Thermo Fisher Scientific, Inc.) according to the manufacturer's protocol. The relative mRNA levels were calculated based on housekeeping gene $\beta$-actin and normalized to BMMSCs according to their $\Delta \Delta \mathrm{Cq}$ values (12). The following primers were used: Crx, 5'-TCACTATT-3' (forward) and 5'-CCTCAC GTGCATACACATCC-3' (reverse); and $\beta$-actin, 5'-TGGCAC CCAGCACAATGAA-3' (forward) and 5'-CTAAGTCATAG TCCGCCTAGAAGCA-3' (reverse). Primers were obtained from Takara Biotechnology Co., Ltd. (Dalian, China).

Western blot analysis. Total protein was extracted with radioimmunoprecipitation assay buffer (Thermo Fisher Scientific, Inc.) containing 1:100 protease inhibitor cocktail. The protein concentration in the extracted lysates was measured using a bicinchoninic acid assay kit (Beyotime Institute of 
Biotechnology, Haimen, China). Proteins (50 $\mu \mathrm{g}$ per sample) were separated by $10 \%$ SDS-PAGE and transferred to a polyvinylidene fluoride membrane (Bio-Rad Laboratories, Inc.). Following blocking with $5 \%$ bovine serum albumin (Gibco; Thermo Fisher Scientific, Inc.) for $1 \mathrm{~h}$ at room temperature, the membranes were incubated with primary antibodies against Crx (1:500; sc-30150; Santa Cruz Biotechnology, Inc., Dallas, TX, USA) and GAPDH (1:1,000; NB100-56875; Novus Biologicals, Ltd., Cambridge, UK) overnight at $4^{\circ} \mathrm{C}$. The immune complexes were incubated for $30 \mathrm{~min}$ at room temperature with horseradish peroxidase-conjugated goat anti-rabbit antibody (1:2,000; BA1055; Wuhan Boster Biological Technology, Ltd., Wuhan, China). Detection was performed using the Western-Light Chemiluminescent Detection system (Bio-Rad Laboratories, Inc.). Blots were analyzed by ImageJ $1.48 \mathrm{v}$ (https://imagej.nih.gov/ij/) and band gray scale was calculated. Intensity was calculated relative to GAPDH.

Immunofluorescence analysis. The PCMs and BMMSCs were either separately cultured or co-cultured on coverslips for 7 days in growth medium, followed by differentiation in induction medium for 21 days, as aforementioned. Cells were rinsed in PBS and fixed in $4 \%$ paraformaldehyde for $15 \mathrm{~min}$ at room temperature. The cells were permeabilized by $0.5 \%$ Triton X-100 (in PBS) for $20 \mathrm{~min}$ at room temperature and then blocked in 5\% normal goat serum (NBP2-23475; Novus Biologicals, Ltd.) for $30 \mathrm{~min}$ at room temperature. Primary rat monoclonal antibodies, including anti-rod photoreceptor rhodopsin (1:200; NBP2-25159; Novus Biologicals, Ltd.), anti-bipolar neurons visual system homebox 2 (CHX10; 1:500; NBP1-84476; Novus Biologicals, Ltd.) and anti-Müller glia heparin sulfate (1:300; MAB2040; EMD Millipore, Billerica, MA, USA) were diluted and incubated with cells overnight at $4^{\circ} \mathrm{C}$. The secondary antibodies (fluorescein isothiocyanate-conjugated) were diluted 1:32 (BA1101 and BA1105; Wuhan Boster Biological Technology, Ltd.) and incubated for $30 \mathrm{~min}$ at room temperature in the dark. Following washing thoroughly with PBS, the coverslip was observed by an inverted fluorescence microscope (Olympus Corporation). The positive rate was determined as the percentage of green cells by ImageJ 1.48v (https://imagej.nih.gov/ij/).

Statistical analysis. All experiments were performed in triplicate and data was presented as the mean \pm standard deviation. One-way analysis of variance followed by the Student-Newman-Keuls test was performed using GraphPad Prism 6.0 (GraphPad Software Inc., La Jolla, CA, USA) to compare different groups. $\mathrm{P}<0.05$ was considered to indicate a statistically significant difference.

\section{Results}

Cell growth observation. PCMs formed numerous colonies under inverted microscope observation (Fig. 1A). BMMSCs exhibited a conventional spindle-like shape (Fig. 1B). When co-cultured together, numerous elongated cells were observed and the cell margin was blurred (Fig. 1C).

Alteration of proliferation. Generally, all groups exhibited a continuous increase in the optical density with the incubation

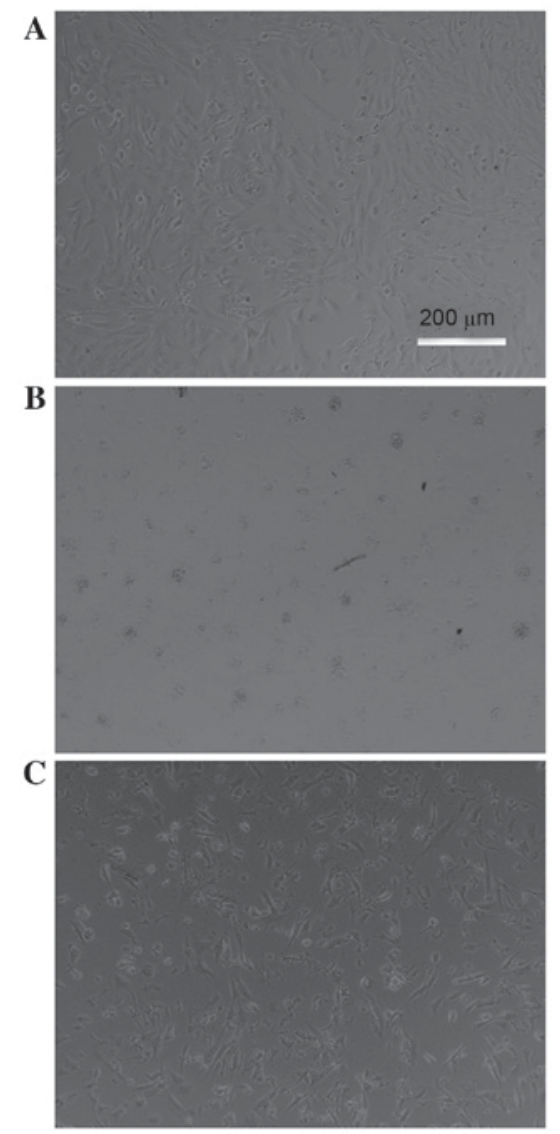

Figure 1. Growth observation under inverted microscope. (A) Pure BMMSCs (B) Pure PCMs. (C) Direct co-culture of BMMSCs and PCMs. All cells were incubated for 3 days in low serum medium to perform observations. The pure BMMSCs exhibited a conventional spindle-like shape while PCMs formed sphere colonies. When co-cultured together, numerous elongated cells were observed and the cell margin was blurred. Scale bar is the same for all images. BMMSCs, bone marrow mesenchymal stem cells; PCMs, pigmented cells from the ciliary margin.

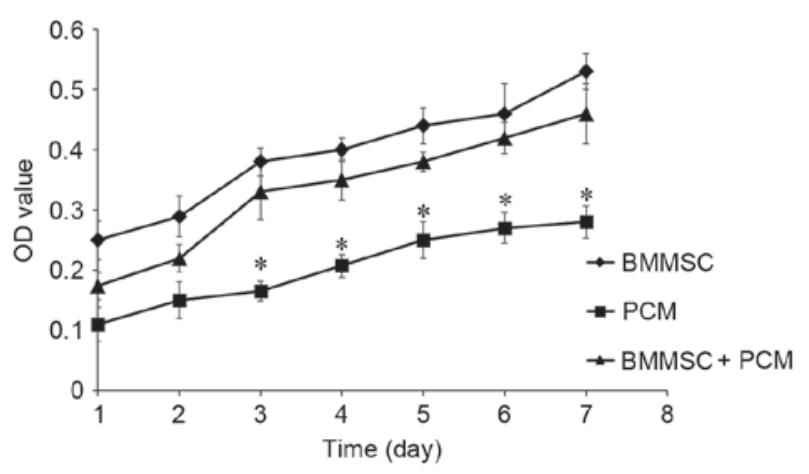

Figure 2. Proliferation curve of BMMSC, PCM and BMMSC + PCM groups. The viability of different cell cultures was measured by MTT assay continuously for 7 days and the OD of each group on each day was recorded to plot the curve. BMMSCs, bone marrow mesenchymal stem cells; PCMs, pigmented cells from the ciliary margin; $\mathrm{OD}$, optical density. ${ }^{*} \mathrm{P}<0.05$, vs. BMMSC and BMMSC + PCM

time (Fig. 2). PCMs had the lowest cell viability throughout the 7 days, while BMMSCs had the highest (Fig. 2). PCM levels were statistically lower compared with the other 2 groups apart from on the first 2 days ( $\mathrm{P}<0.05$; Fig. 2 ). Co-cultured cells had optical density values marginally lower 
A

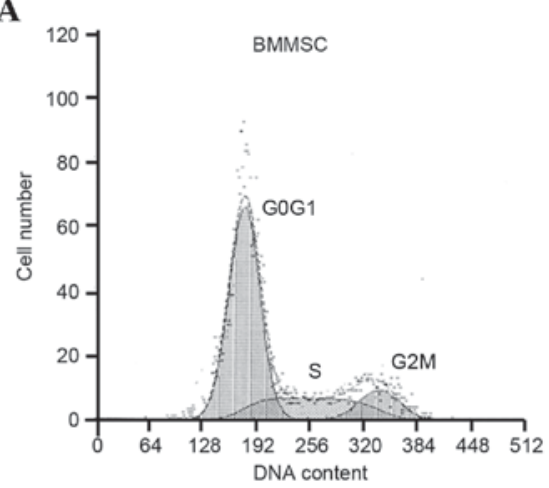

C

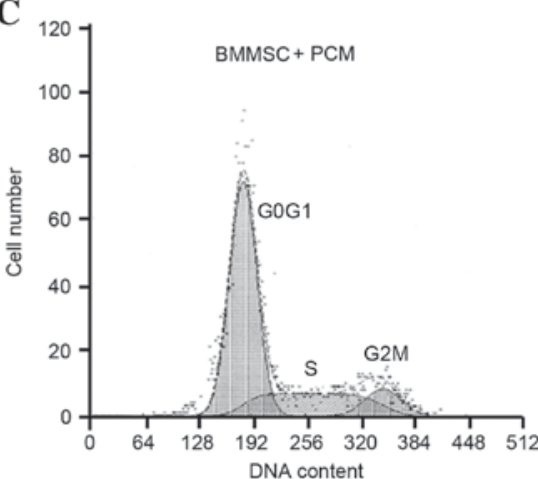

B

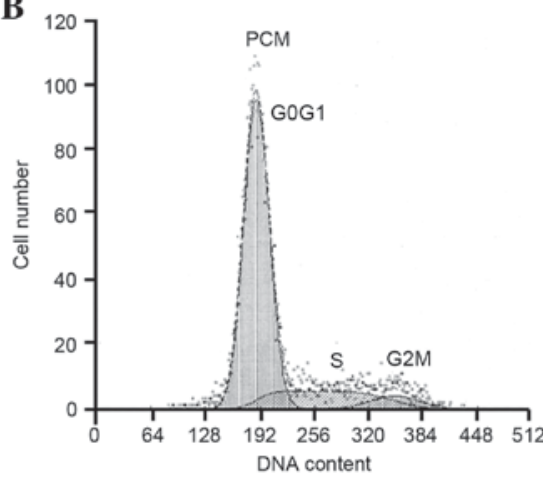

D

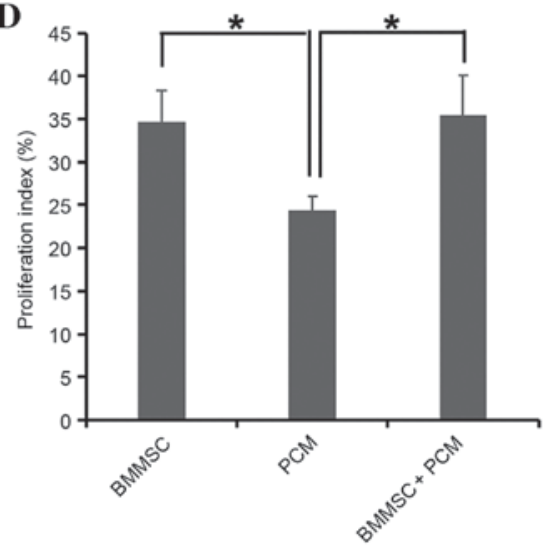

Figure 3. Cell cycle analysis by flow cytometry 3 days post-cell culture. (A) Pure BMMSCs. (B) Pure PCMs. (C) Direct co-culture of BMMSCs and PCMs (D) Proliferation index was calculated as the percentage of $\mathrm{S}+\mathrm{G} 2 / \mathrm{M}$. " $\mathrm{P}<0.05$, as indicated on the figure. BMMSCs, bone marrow mesenchymal stem cells; PCMs, pigmented cells from the ciliary margin.

A

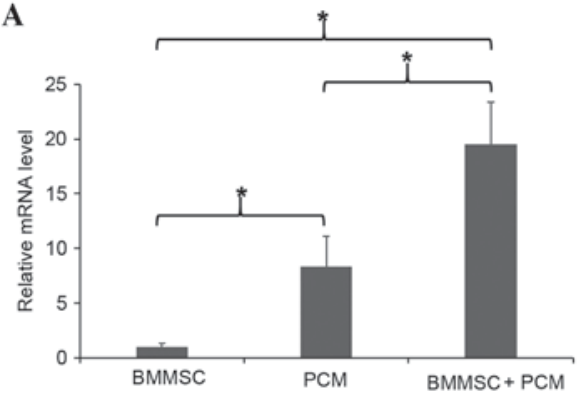

B

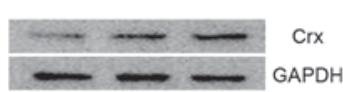

C

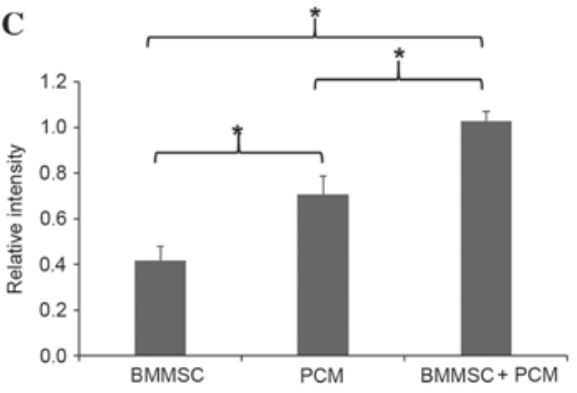

Figure 4. Expression of $\mathrm{Crx}$ in different cell cultures following 21 days of induction. (A) mRNA level of Crx measured by quantitative polymerase chain reaction analysis. (B) Protein levels of Crx detected by western blot analysis. (C) Gray scale analysis of western blot results by ImageJ software. ${ }^{*} \mathrm{P}<0.05$. Crx, cone-rod homeobox; BMMSCs, bone marrow mesenchymal stem cells; PCMs, pigmented cells from the ciliary margin. compared with BMMSCs, however, this was not significant (P>0.05; Fig. 2).

Cell cycle analysis. Cell cycle analysis was performed 3 days post-cell culture. The number of cells at G1 phase in BMMSCs was higher compared with PCMs (Fig. 3A and B). In co-cultured cells, G1 phase number was between that of the PCM and BMMSC groups (Fig. 3C). The proliferation index of PCMs was significantly lower compared with BMMSC and BMMSC + PCM groups $(\mathrm{P}<0.05$; Fig. 3D), and no statistical difference was observed between BMMSC and BMMSC + PCM groups ( $\mathrm{P}>0.05$; Fig. 3D).

Crx gene expression. The gene expression of photoreceptor-specific homeobox gene, $\mathrm{Crx}$, was measured by RT-qPCR after induction for 21 days. The PCM group expressed significantly higher levels ( $\sim 10$ fold higher) of Crx compared with the BMMSC group ( $\mathrm{P}<0.05$; Fig. 4A). Notably, Crx expression in co-cultured cells was significantly upregulated ( $\sim 2$ fold higher) compared with the PCM group ( $\mathrm{P}<0.05$; Fig. 4A). Western blot analysis revealed similar results; $\mathrm{Crx}$ protein expression in PCM was significantly higher compared with the BMMSC group and expression in co-cultured cells was significantly higher compared with the PCM group ( $\mathrm{P}<0.05$; Fig. 4B).

Immunofluorescence staining. To further confirm the retina differentiation, RSC-associated markers were stained. All markers that were investigated were expressed in the observed 

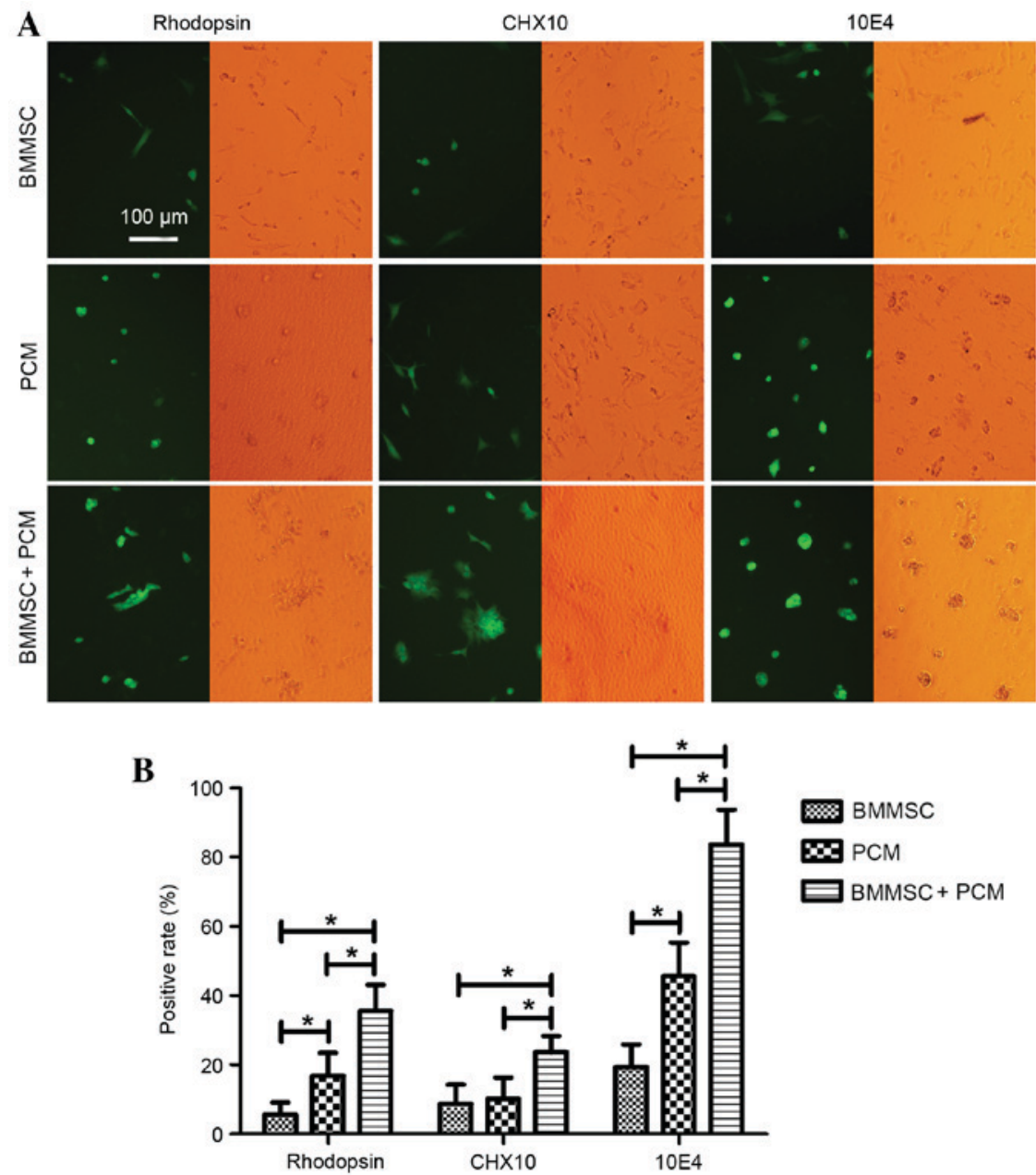

Figure 5. Immunofluorescence analysis of rhodopsin, visual system homeobox 2 and heparin sulfate following 21 days of induction. (A) Images were taken by inverted microscope in the fluorescence channel and light channel. (B) Quantification of immunofluorescence results by ImageJ software. "P<0.05. Rho1D4, rhodopsin; CHX10, visual system homeobox 2; 10E4, heparin sulfate; BMMSCs, bone marrow mesenchymal stem cells; PCMs, pigmented cells from the ciliary margin.

cells with different positive ratios (Fig. 5A). Specifically, as illustrated in Fig. 5B, the percentage of rhodopsin-positive cells in the groups were 5.63 $\pm 3.52 \%$ (BMMSC), $16.80 \pm 6.66 \%$ (PCM) and $35.50 \pm 7.66 \%$ (BMMSC + PCM). The percentage of CHX10-positive cells in different groups were $8.72 \pm 5.58 \%$ (BMMSC), $10.12 \pm 6.20 \%$ (PCM) and $23.60 \pm 4.66 \%$ (BMMSC + PCM). The percentage of heparin sulfate-positive cells in different groups were $19.30 \pm 6.58 \%$ (BMMSC), $45.50 \pm 9.83 \%(\mathrm{PCM})$ and $83.60 \pm 10.12 \%(\mathrm{BMMSC}+\mathrm{PCM})$.

\section{Discussion}

The retina senses light and sends signals to the brain through the optical nerve to produce an image. The regeneration of the retina is problematic once the damage has been caused by glaucoma. However, although the central nerve is considered to be difficult to regenerate, it may be possible with the optical nerve. Numerous studies have confirmed that the optical nerve is able to regenerate under certain circumstances and that stem cells have a major role in the regeneration $(2,13)$. Stem cells can be isolated from various human tissues and it may be deduced that stem cells prefer to differentiate into the tissue where it originates from, despite their multipotent potential.
For example, BMMSCs have a strong ability for osteogenesis, while adipose-derived stem cells easily undergo adipogenic differentiation (14). In addition, the present study confirmed this as the PCM group exhibited greater retinal differentiation compared with the BMMSC group.

Another important factor during stem cell engineering is the cell number. A sufficient cell number is not only essential to acquire sufficient differentiated cells, it is also important for cell survival (15). Although PCMs exhibit high retinal differentiation potential, the proliferation ability of PCMs is limited. In the present study, the co-culture of PCMs and BMMSCs increased cell proliferation rate compared with PCMs and exhibited no significant difference compared with BMMSCs alone. In addition, co-cultured cells exhibited higher retinal marker expression compared with PCMs alone. The results indicated that direct co-culture of PCMs and BMMSCs may provide a novel stem cell group that has improved proliferation and differentiation ability, which may be more suitable for RGC regeneration.

The mechanism of fusion of PCMs and BMMSCs has not been investigated in the present study; however, the most probable explanation ought to be the 'cell fusion' phenomenon (16). In fact, previous studies have misinterpreted this 
phenomenon as 'transdifferentiation' $(16,17)$. Adult stem cells are able to spontaneously fuse with embryonic stem cells and take on their characteristics $(18,19)$. Not only embryonic stem cells, MSCs may also fuse with and differentiate into other cell types, including airway epithelial cells (20) and gastrointestinal epithelial cells (21). Although the present study has not confirmed the fusion phenomenon, it is reasonable to deduce that BMMSCs fused with PCMs in the co-culture procedure and the newly formed cells may be a more useful type of RSC for retina regeneration.

\section{References}

1. Casson RJ, Chidlow G, Wood JP, Crowston JG and Goldberg I: Definition of glaucoma: Clinical and experimental concepts. Clin Exp Ophthalmol 40: 341-349, 2012.

2. Benowitz LI and Yin Y: Optic nerve regeneration. Arch Ophthalmol 128: 1059-1064, 2010.

3. Diekmann H and Fischer D: Glaucoma and optic nerve repair. Cell Tissue Res 353: 327-337, 2013

4. Reh TA and Fischer AJ: Retinal stem cells. Methods Enzymol 52-73, 2006.

5. Tropepe V, Coles BL, Chiasson BJ, Horsford DJ, Elia AJ, McInnes RR and van der Kooy D: Retinal stem cells in the adult mammalian eye. Science 287: 2032-2036, 2000.

6. Ramsden CM, Powner MB, Carr AJ, Smart MJ, da Cruz L and Coffey PJ: Stem cells in retinal regeneration: Past, present and future. Development 140: 2576-2585, 2013.

7. Auletta JJ, Deans RJ and Bartholomew AM: Emerging roles for multipotent, bone marrow-derived stromal cells in host defense. Blood 119: 1801-1809, 2012.

8. Xu W and Xu GX: Mesenchymal stem cells for retinal diseases. Int J Ophthalmol 4: 413-421, 2011.

9. Chiou SH, Kao CL, Peng CH, Chen SJ, Tarng YW, Ku HH, Chen YC, Shyr YM, Liu RS, Hsu CJ, et al: A novel in vitro retinal differentiation model by co-culturing adult human bone marrow stem cells with retinal pigmented epithelium cells. Biochem Biophys Res Commun 326: 578-585, 2005.
10. Gómez-Lechón MJ and Tolosa L: Hepatogenic differentiation: Comparison between adipose tissue-derived stem cells and bone marrow mesenchymal stem cells. Stem Cells and Cancer Stem Cells 10: 45-57, 2013.

11. Sylvester PW: Optimization of the tetrazolium dye (MTT) colorimetric assay for cellular growth and viability. Methods Mol Biol 716: 157-168, 2011.

12. Livak KJ and Schmittgen TD: Analysis of relative gene expression data using real-time quantitative PCR and the 2(-Delta Delta C(T)) method. Methods 25: 402-408, 2001.

13. Dahlmann-Noor A, Vijay S, Jayaram H, Limb A and Khaw PT: Current approaches and future prospects for stem cell rescue and regeneration of the retina and optic nerve. Can J Ophthalmol 45: 333-341, 2010.

14. Strioga M, Viswanathan S, Darinskas A, Slaby O and Michalek J: Same or not the same? Comparison of adipose tissue-derived versus bone marrow-derived mesenchymal stem and stromal cells. Stem Cells Dev 21: 2724-2752, 2012.

15. Mahla RS: Stem cells applications in regenerative medicine and disease therapeutics. Int J Cell Biol 2016: 6940283, 2016.

16. Wurmser AE and Gage FH: Stem cells: Cell fusion causes confusion. Nature 416: 485-487, 2002.

17. Eisenberg LM and Eisenberg CA: Stem cell plasticity, cell fusion, and transdifferentiation. Birth Defects Res C Embryo Today 69: 209-218, 2003.

18. Ying QL, Nichols J, Evans EP and Smith AG: Changing potency by spontaneous fusion. Nature 416: 545-548, 2002.

19. Terada N, Hamazaki T, Oka M, Hoki M, Mastalerz DM, Nakano Y, Meyer EM, Morel L, Petersen BE and Scott EW: Bone marrow cells adopt the phenotype of other cells by spontaneous cell fusion. Nature 416: 542-545, 2002.

20. Spees JL, Olson SD, Ylostalo J, Lynch PJ, Smith J, Perry A, Peister A, Wang MY and Prockop DJ: Differentiation, cell fusion, and nuclear fusion during ex vivo repair of epithelium by human adult stem cells from bone marrow stroma. Proc Natl Acad Sci USA 100: 2397-2402, 2003.

21. Ferrand J, Noël D, Lehours P, Prochazkova-Carlotti M, Chambonnier L, Ménard A, Mégraud F and Varon C: Human bone marrow-derived stem cells acquire epithelial characteristics through fusion with gastrointestinal epithelial cells. PLoS One 6: e19569, 2011. 